\title{
(2) OPEN ACCESS \\ Complex advance care planning interventions for chronic serious illness: how do they work: a scoping review
}

\author{
Julie Stevens (10) ,1,2 Luc Deliens, 1,2,3 Peter Pype, ${ }^{1,3}$ Aline De Vleminck, ${ }^{1,2}$ \\ Koen Pardon ${ }^{1,2}$
}

- Additional supplemental material is published online only. To view, please visit the journal online (http://dx.doi. org/10.1136/bmjspcare-2021 003310).

${ }^{1}$ Vrije Universiteit Brussel (VUB) \& Ghent University, End-of-life Care Research Group, Brussels, Belgium

${ }^{2}$ Department of Family Medicine and Chronic Care, Vrije Universiteit Brussel (VUB), Brussels, Belgium

${ }^{3}$ Department of Public Health and Primary Care, Ghent University, Ghent, Belgium

\section{Correspondence to}

Ms Julie Stevens, Vrije Universiteit Brussel (VUB) \& Ghent University, End-of-life Care Research Group, Brussels, Belgium;

Julie.joseph.stevens@vub.be

Received 4 August 2021

Accepted 2 September 2021

D Check for updates

(C) Author(s) (or their employer(s)) 2021. Re-use permitted under CC BY-NC. No commercial re-use. See rights and permissions. Published by BMJ.

To cite: Stevens J, Deliens L, Pype P, et al. BMJ Supportive \& Palliative Care Epub ahead of print: [please include Day Month Year]. doi:10.1136/

bmjspcare-2021-003310

\section{ABSTRACT}

Context Advance care planning (ACP) interventions have the potential to improve outcomes for patients with chronic serious illness. Yet the rationale for outcome choices and the mechanisms by which outcomes are achieved are not always clear.

Objectives To identify and map proposed mechanisms on how complex ACP interventions can impact outcomes for patients with chronic serious illness and to explore factors that might explain intervention outcomes.

Methods This is a scoping review of randomised controlled trials of complex ACP interventions for patients with chronic serious illness which explicitly stated the mechanism(s) by which the intervention was thought to work. We searched six databases and hand-searched key journals and reference lists.

Results Inclusion yielded 16 articles. Inclusion procedures and mapping of mechanisms and outcomes indicated that causality between components and outcomes was not always clearly described. Tailoring intervention content to patients' needs was linked to the greatest number of different outcome categories, while promoting competence and confidence to engage in ACP was most often explicitly linked to a primary outcome. Three main factors which might have affected intended outcomes were identified: participant characteristics, such as illness experience or cultural differences; the setting of implementation; or methodological limitations of the study.

Conclusion Findings highlighted two main points of consideration for future ACP intervention studies: the need for clearly stated logic in how interventions are expected to impact primary outcomes and the importance of considering how an intervention may function for patients with chronic serious illnesses within a specific setting.

\section{Key messages}

What was already known?

- Examining how advance care planning (ACP) interventions are currently proposed to affect outcomes can guide expectations of what ACP can achieve.

What are the new findings?

- Building skills and confidence was most often used to explicitly state how the intervention would affect outcomes.

- Contextual factors considered a priori mainly related to sociocultural factors and racial disparities, and other factors were considered in light of the results obtained.

What is their significance?

- Intervention rationales are identified, but further clarity is needed regarding how components operate.

- Participants, setting and study design effects should be considered a priori.

\section{INTRODUCTION}

Advance care planning (ACP) refers to a process which 'enables individuals to define goals and preferences for future medical treatment and care, to discuss these goals and preferences with family and health-care providers, and to record and review these preferences if appropriate'. ${ }^{2}$ This can, but not necessarily must, entail or result in the completion of an advance directive (AD) specifying care wishes, which can be used in the event the patient loses decisional capacity. It can also involve the appointment of a surrogate decision maker (SDM) who makes decisions about medical care in the patient's stead. By communicating with health professionals, informal caregivers, family members and loved ones, patients engaging in ACP can make their wishes 
for future health care known. Additionally, ACP can help patients and their SDM be better prepared to make the best possible in-the-moment care decisions together with clinicians. ${ }^{2}$

Studies of interventions designed to implement or improve ACP, as well as reviews of the ACP research literature, demonstrate a large degree of heterogeneity in outcomes and evidence of effectiveness, and suggest that complex interventions may be more effective in meeting patient preferences for end-oflife care. ${ }^{3-7}$ The spectrum of outcomes ranges from proximal outcomes, such as ACP engagement, to distal outcomes such as concordance of care with patient wishes. ${ }^{8}$ For many of these outcomes, reviews report mixed findings. ${ }^{3-5} 79$ Most recently, a scoping review of ACP randomised controlled trials (RCTs) mapped the outcomes of interventions according to a standardised ACP Outcome Framework. This review found a greater percentage of positive results for outcome domains related to processes (such as knowledge) and actions (such as completion of an $\mathrm{AD}$ ) than for quality of care, health status and healthcare utilisation. As a result, the reviewers recommended further research to tailor interventions to specific contexts and to set appropriate expectations of ACP outcomes. ${ }^{10}$ Taken together, this highlights the current state of the scientific discourse around ACP in terms of its conceptualisation and its goals. ${ }^{11-14}$ Indeed, questions have been posed about whether ACP has the capacity to address what has been defined by a Delphi panel as its most important outcome, namely goal-concordant care, leading to discussions regarding what the focus of ACP research ought to be. ${ }^{15-17}$

The workings of ACP as a process are complex, ${ }^{18} 19$ but this complexity may not always be addressed in the research literature and may be overlooked when interventions and outcomes are considered discretely. To set appropriate expectations for ACP interventions, it is important to consider not only the content of an intervention, but also how the intervention is expected to lead to the outcomes of interest. ${ }^{20} \mathrm{~A}$ recent review of ACP interventions for patients with cancer indicated that studies propose different mechanisms by which ACP interventions are expected to affect outcomes, but that not every paper does so in equal detail. ${ }^{21}$ This implies that adequate attention for the rationale ${ }^{22}$ of ACP interventions may be lacking, at least in the reporting of these interventions. In the middle of a debate where the benefit of continuing ACP research is coming into question, ${ }^{16}$ this is especially concerning. In a response to Dr Morrison's notes from the editor, ${ }^{16}$ Montgomery et al. ${ }^{23}$ call for further work to develop evidence-based conceptual models of ACP. This response further notes that the intervention logic must also be considered within the system where the intervention is implemented. To our knowledge, however, no review of ACP interventions exists for chronic serious illness that specifically investigates not only intervention components and outcomes, but also the mechanisms by which the intervention is hypothesised to affect said outcomes-that is, what do authors expect the intervention will achieve and how? Neither has a review examined whether and how the authors of these articles explain the study findings that were obtained-for example, if the intervention did not achieve the expected results, is this due to factors intrinsic to the intervention; do systemic factors preclude intervention impact; or should the hypothesised mechanism be re-evaluated to more accurately reflect what ACP can accomplish?

Insights in these fields will aid the development of new interventions to facilitate ACP or the refining of existing initiatives. This is made possible by first identifying pathways from interventions to outcomes via hypothesised mechanisms. Then we can assess which pathways yield positive results, while also highlighting contextual and implementation factors that should be considered. Altogether, this allows us to identify gaps in the current research at different 'links' within this 'chain'.

The purpose of the present scoping review was to identify which mechanisms are proposed to explain how complex ACP interventions, tested for effectiveness through an RCT, are expected to impact outcomes for patients with serious illness, to establish the factors authors refer to in order to explain the study findings, and to map the available evidence.

The research questions can be summarised as follows:

- What are the core components of the intervention?

- Which primary outcome(s) is/are chosen to evaluate the effectiveness of the intervention?

- What is the mechanism by which authors propose the intervention will work to generate change in outcomes?

- What are the results for the primary outcome(s)?

- Which factors contributed to intervention success or failure to affect the primary outcome(s), according to the authors?

\section{METHODS}

\section{Study design}

To address the aims of this study, a scoping review design was considered most appropriate. ${ }^{24}$ A scoping review is a process of mapping and describing the existing literature, which can be undertaken when the area of study in question is complex ${ }^{25}$ and/or heterogeneous in methods or in discipline. ${ }^{26}$ This scoping review was conducted according to the methodological framework set forth by Arksey and O'Malley ${ }^{25}$ and the additional recommendations by Levac et al. ${ }^{27}$ The framework recommends the following steps: (1) identifying the research question; (2) identifying relevant studies; (3) study selection; (4) charting the data; and (5) collating, summarising and reporting the results. The Preferred Reporting Items for Systematic Reviews and Meta-Analyses extension for Scoping Reviews 
checklist (PRISMA-ScR) ${ }^{26}$ was followed for writing the present review report.

\section{Search strategy}

We conducted an online search of the following databases: PubMed, PsycINFO, MEDLINE, Embase, Cochrane Central Register of Controlled Trials (CENTRAL) and the Cumulative Index to Nursing and Allied Health Literature (CINAHL). Key terms, Medical Subject Headings (MeSH), Emtree headings and CINAHL headings were used in their applicable databases. The search was first conducted in March 2020 using a search strategy informed by terms and keywords used in existing systematic reviews of ACP. $^{3-6}$ This strategy underwent piloting to ensure the search was sensitive enough to capture key articles, yet specific enough to avoid capturing large numbers of irrelevant articles (see online supplemental figure 1 for an example search strategy). The search was last repeated on 18 November 2020 to identify additional articles that may have been published since the initial search. We included studies that were published in the English language from 1 January 2010 to the date of the last search. Filters were applied for publication type (journal articles, articles in press) where possible.

The references of key articles were screened for additional studies to include. We also hand-searched the most recent issues of the following key journals: Journal of Palliative Care, Journal of Palliative Medicine, Journal of Pain and Symptom Management, Journal of the American Medical Association, BMJ Supportive \& Palliative Care, and Palliative Medicine.

\section{Eligibility criteria}

Papers were considered for inclusion based on the criteria specified in table 1 .

A primary determinant of intervention complexity was the number of components in the intervention, which could be delivered separately or as a package. Interventions with two or more components were considered complex. Interventions consisting of one facilitated discussion were also considered complex due to the flexibility permitted and the training required of the person facilitating the discussion. ${ }^{20}$
The study introduction and methods sections were read closely multiple times to ascertain whether the study explicitly states or describes the mechanism(s) by which the intervention was expected to generate changes in outcomes.

Methodological quality was not considered an exclusion criterion as we sought to provide an overview from RCTs of complex ACP interventions for chronic serious illness.

\section{Selection of sources of evidence}

All retrieved articles were uploaded to Zotero reference manager. Duplicates of retrieved records were removed. Titles and abstracts of all identified articles were screened against the eligibility criteria by JS using a standardised form. Articles identified as potentially relevant were retrieved in full. Full-text screening against the eligibility criteria was completed by JS. During both steps of the screening process, cases of doubt were checked by a second researcher (ADV). During title and abstract screening, if there was unresolved ambiguity, the article was retained for fulltext review. In the case of ambiguity in the full-text screening step, a third researcher (KP) was consulted for discussion to achieve consensus.

\section{Data charting process}

The following characteristics of the study were extracted using a standardised data charting sheet, which was developed through iterative discussions between the authors: study authors, publication year, country, study setting and sample characteristics. Data extracted regarding the study design included conceptual model or theory used (if any), core intervention components, control condition, duration of the intervention, primary outcome(s), mechanisms and primary findings. Each article was examined for the implications of the results for the proposed mechanism(s) and/ or factors which were considered to have impacted the results (eg, contextual factors). Relevant text excerpts were extracted. Two authors (JS and ADV) crosschecked the data from a small sample of studies to achieve consensus regarding the extracted text, after

Table 1 Inclusion and exclusion criteria

\begin{tabular}{|c|c|}
\hline Inclusion criteria & Exclusion criteria \\
\hline $\begin{array}{l}\text { Concerns a peer-reviewed article. } \\
\text { Reported in English. } \\
\text { Of primary research. } \\
\text { Reporting on the quantitative primary outcome(s). } \\
\text { Of a randomised controlled trial. } \\
\text { Of a complex advance care planning intervention. } \\
\text { For chronic serious illness. } \\
\text { In a sample of adults ( }>18 \text { years old). } \\
\text { Where the mechanism(s) by which the intervention is expected to generate } \\
\text { changes in outcomes is/are described. }\end{array}$ & $\begin{array}{l}\text { Published prior to } 1 \text { January } 2010 \text {. } \\
\text { Does not state which outcome is considered the primary outcome. } \\
\text { Reports feasibility and/or satisfaction outcomes only. } \\
\text { Reports on a secondary analysis of a randomised controlled trial only. } \\
\text { Reports on a trial where advance care planning (ACP) is embedded in a broader } \\
\text { intervention, such as palliative care interventions that also include pain or } \\
\text { symptom management, so that it is not possible to distinguish the discrete } \\
\text { effects of the ACP component. } \\
\text { Reports on psychiatric advance directives and/or crisis planning. } \\
\text { Reports a study protocol only. }\end{array}$ \\
\hline
\end{tabular}




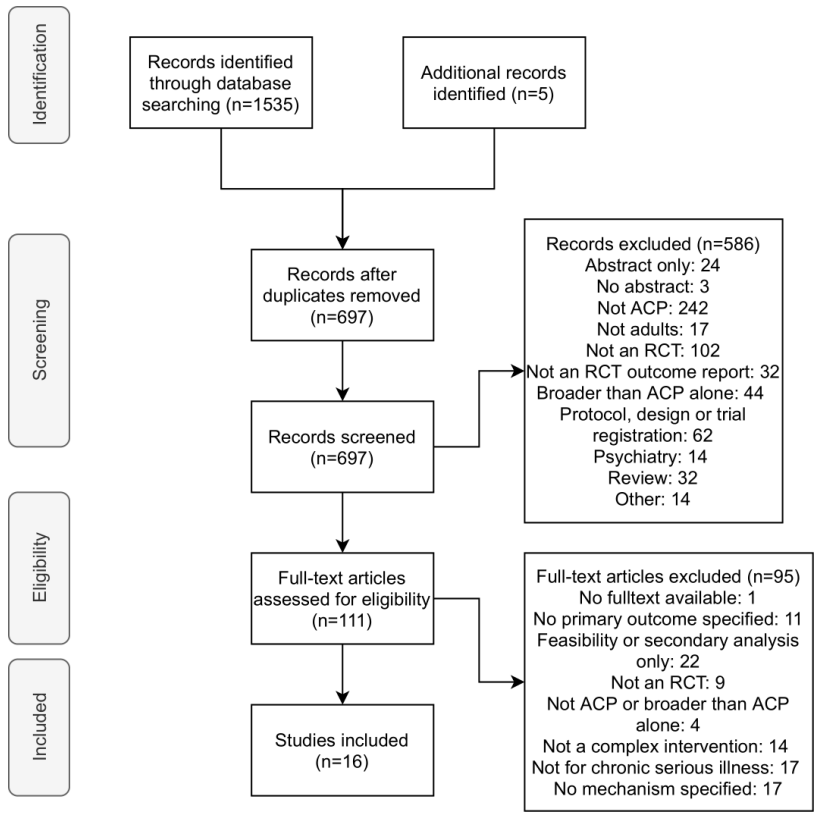

Figure 1 PRISMA flow chart of search results. ACP, advance care planning; PRISMA, Preferred Reporting Items for Systematic Reviews and Meta-Analyses; RCT, randomised controlled trial.

which one author (JS) proceeded with the remaining charting.

\section{Synthesis of results}

After data extraction, we used descriptive statistics to describe the included studies. Relevant excerpts were imported into NVivo for qualitative analysis. In the first step, open codes were applied to the extracted text regarding intervention components, mechanisms and factors impacting the effect of the intervention on the primary outcome(s). The codes were then combined into descriptive themes. Mechanisms and the outcomes they were expected to generate were mapped in order to indicate which retained articles specified links between mechanisms and outcomes.

\section{RESULTS}

Of 1535 articles identified, 16 met the eligibility criteria and were retained for analysis (figure 1).

\section{Study characteristics}

The characteristics of the retained studies can be found in online supplemental table 1.

Ten studies were conducted in the USA, two each in the Netherlands and Australia, and one each in Belgium and Northern Ireland. Participants included patients with cancer, dementia, geriatric frailty, endstage renal disease, heart failure, HIV and comorbid chronic serious illnesses. Nine studies included dyads of patients and a family carer or SDM.

\section{Intervention components}

Of the 16 studies retained (online supplemental table 1), the largest portion (11 studies) made use of an interview or conversation to address the topic of ACP. ${ }^{28-38}$ Conversations were led by facilitators including (trained) nurses, ${ }^{28} \quad 29 \quad 32 \quad 34-36 \quad 38$ psychologists, ${ }^{31} 32{ }^{37}$ social workers ${ }^{31-33}$ or graduate students. ${ }^{31}$ Five conversation interventions also included the provision of conversation summaries and/or ADs completed during the conversation to the healthcare provider (general practitioner or treating physician) or placement of documentation in the patient's health record. ${ }^{2829} 313236$

Eight studies made use of an $\mathrm{AD}$ or goals of care form, which was presented for the patient to read or completed during an interview or discussion. $^{28} 313234366^{39-41}$ Six studies used informational material in online repository, pamphlet or video format. ${ }^{34-41}$ Four studies used question prompts and conversation openers for patients or communication tips for health providers, ${ }^{29} 384142$ with two of these studies also including instructions for health providers to facilitate the discussion or endorse use of question prompts. ${ }^{29} 38$ Three studies used an interactive decision aid in website format for patients. ${ }^{39-41}$ Two interventions used interactive educational workshops for general practitioners or nursing home staff. ${ }^{42}$ One intervention provided ACP education to participants, but the content of the education was not specified. ${ }^{33}$

What are the primary outcomes and by which mechanism do authors propose they are achieved?

The outcomes and the mechanisms by which they were proposed to be achieved are mapped in figure 2 .

In total, nine types of primary outcomes were identified: decisional conflict, dyad congruence, $\mathrm{AD}$ or Do-Not-Resuscitate (DNR) order completion, psychospiritual well-being, quality of life, number of conversations/consultations, content of discussions with health providers, patient activation and place of death.

Seven descriptive themes were identified to describe the mechanisms by which interventions were proposed to affect the primary outcome(s): (1) tailoring the intervention to the patient recipient (their coping style, health literacy, desire for information, beliefs and misconceptions) to make the intervention more understandable and acceptable; (2) educating patients and carers/surrogates about the course of illness, treatment options and styles of shared decision making in order to facilitate better understanding of these topics; (3) encouraging active involvement of carers/surrogates and health providers in ACP conversations to promote a shared understanding of the patient's values and preferences; (4) exploring the patient's illness beliefs, preferences and values in a systematic way to give the patient an opportunity to reflect on these topics; (5) increasing the degree to which patients, family carers/surrogates and health providers recognise the importance of ACP; (6) addressing the patient's need for social support in their decision making and ACP 


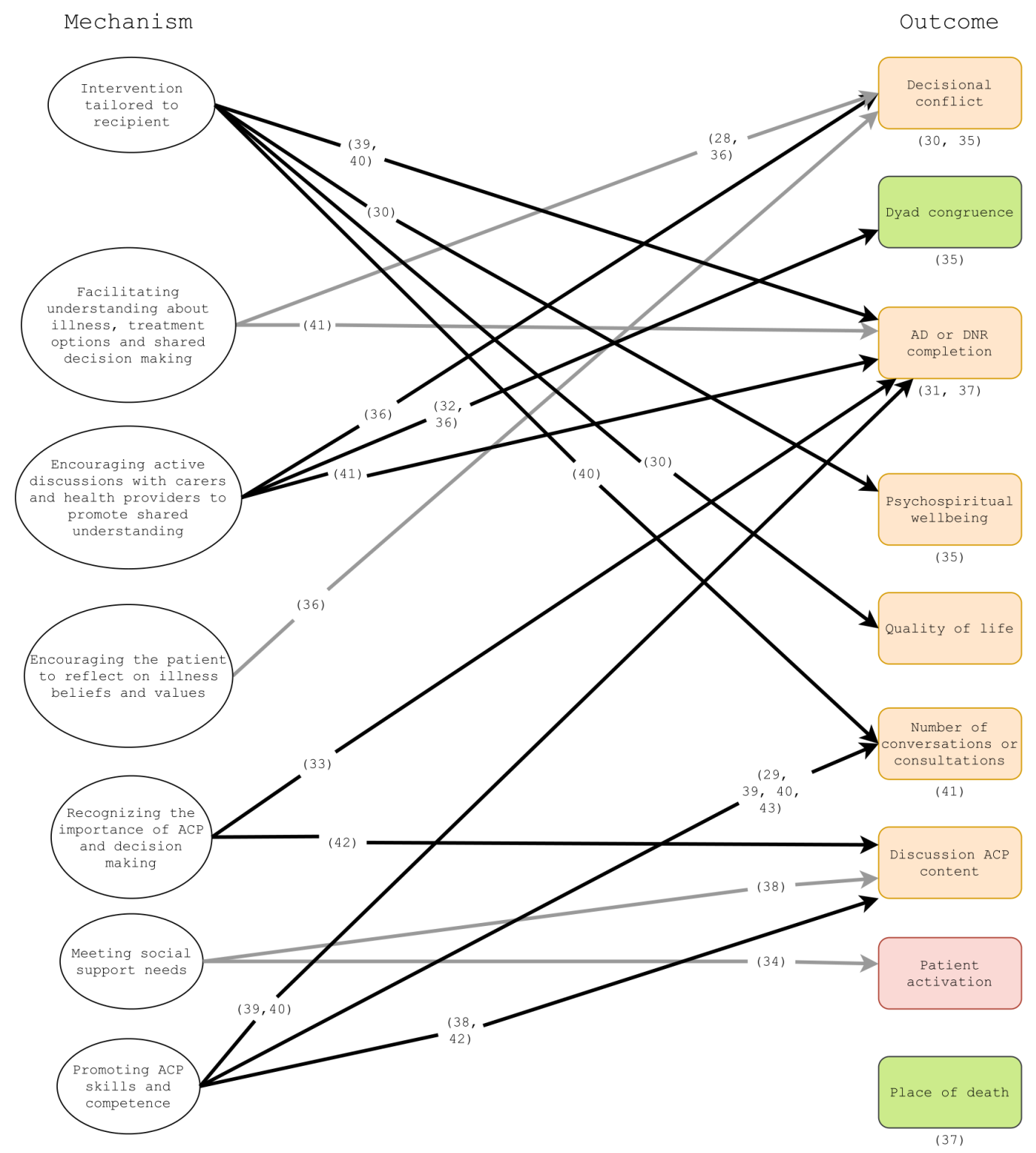

Figure 2 Linkage of mechanisms to outcomes. In this figure, references where linkages between mechanisms and outcomes were made are illustrated using arrows. Where an outcome is described but not how it is expected to be achieved, the reference is placed under the outcome cell. Green outcome cells: positive findings; red outcome cells: negative findings; orange outcome cells: mixed findings. ACP, advance care planning; AD, advance directive; DNR, Do-Not-Resuscitate order

behaviour; and (7) promoting patient and health provider skills, competence and confidence necessary to have ACP conversations.

Studies ranged from referring to a single mechanism (eg, facilitating family carer understanding of the course of dementia and different therapeutic options in order to reduce decisional uncertainty $)^{28}$ to several mechanisms (eg, attending to health literacy and other individual differences, building skills to engage in ACP and encouraging the inclusion of family in the process). ${ }^{40}$

Tailoring the delivery style or content of the intervention to the particular needs of the patient recipient, such as by matching intervention delivery to the culture of the patient population, responding to the patient's coping style or by attending to limited health literacy, ${ }^{303940}$ was the mechanism linked to the greatest variety of outcome domains. When information was presented in a personalised way, it was anticipated to be more understandable and acceptable to the patient, and maximising the prevalent coping strategies of patients allowed for a strengths-based approach in one intervention. ${ }^{30}$ This tailoring was expected to increase the number of conversations patients then conducted about ACP, their completion of ADs, ${ }^{39} 40$ and emotional and health-related outcomes. ${ }^{30}$

Promoting the skills, competence and confidence necessary to participate in ACP conversations, both for patients and health providers, was referred to in the greatest number of studies which stated how the primary outcome was expected to be achieved. It was considered a mechanism for effecting change in the 
number of conversations conducted about ACP and end-of-life care, ${ }^{293940} 43$ for improving AD completion rates, ${ }^{39} 40$ and for increasing active participation and shared decision making during ACP conversations. ${ }^{38} 42$

\section{What are the findings for the primary outcomes?}

An overview of findings for the primary outcomes can be found in online supplemental table 2. Dyad congruence was the only primary outcome for which a positive result was reported across multiple studies. ${ }^{32} 3536$ One study, using a pamphlet and a discussion with a psychologist to help the patient reflect on their values towards end-of-life care, found a reduced likelihood of hospital death. ${ }^{37}$

An intervention including informational leaflets and an ACP conversation during which an $\mathrm{AD}$ was completed did not increase patient activation. ${ }^{34}$

For all other outcomes, results were mixed (see online supplemental table 2).

\section{What impacts whether or not the intervention achieves the desired outcome?}

After coding the factors through which authors explain intervention findings, as well as the success or failure of the intervention to affect the primary outcome(s), three overarching themes were identified: participant factors, implementation factors and methodological factors.

Participant factors include both individual characteristics as well as possible cultural differences. Five studies evaluated an intervention in diverse or non-white populations; these studies all made a priori considerations of the influence of racial disparities and their impact on access to quality end-of-life care. ${ }^{31} 33353640$ According to one study, treating the family as an asset to the ACP process through rapport-building communication can help an intervention align with African American participants' needs. ${ }^{31}$ Minority populations such as Latinos may also face language barriers, which limit access to end-of-life communication. One study reported that, additionally, Latinos may not want to burden family members with distressing issues such as end-of-life conversations. ${ }^{33}$ Attending to barriers such as those posed by language and health literacy can increase the likelihood of intervention success. ${ }^{40}$

The authors of six studies reflected on the impact of the patient's illness experience on study outcomes. Physical pain or discomfort from treatments may reduce the likelihood that a patient engages with the intervention. ${ }^{334}$ In the case of chronic illness or illnesses with a less-predictable trajectory, patients may see treatment as a fundamental part of their lives or aim to live well within their condition, hindering contemplation of alternative care options. ${ }^{313543}$ When question prompts are used in an intervention, patients who are not exclusively receiving palliative care may find it difficult to ask questions about the end of life, even after prompting. ${ }^{38}$
In order to engage in ACP, patients must be willing to discuss the topic and desire a role in decision making about future care. ${ }^{37}$ When they do not, their engagement with ACP interventions may be limited.

The impact of other patient characteristics was less clear. The effect of age on patient use of online interventions is uncertain. ${ }^{41}$ The differences between heterosexual and homosexual patients in ACP interventions for patients with HIV, also merit further consideration. ${ }^{32}$ Finally, the authors of two studies noted that their samples were predominantly male, but the impact of gender was not further explored. ${ }^{38} 39$

Implementation refers to how the intervention was realised within the study setting. The brief duration of an intervention (eg, one conversation) was noted by two authors as a possible explanation for an absence of effects, as it may not have provided patients enough time to prepare for decision making. ${ }^{30} 35$ The authors of four studies highlighted the importance of engaging the surrogate, caregiver or family ${ }^{29} 323341$; failing to do so may contribute to null results. ${ }^{41}$ The authors of four studies emphasised that for ACP interventions involving health providers to achieve the intended outcomes, providers must be trained in ACP and communication skills so that they can initiate conversations and respond to patients. ${ }^{28} 373843$ Two interventions could not be implemented system-wide ${ }^{34} 42$ and one online intervention was not simple to use. ${ }^{41}$ The context of an RCT also may not fully reflect the context of daily practice. ${ }^{34}$ Finally, the authors of one study considered that findings may have been related to the implementation of ACP within a healthcare context that has a history of avoiding overtreatment, where there may be less to be gained from ACP. ${ }^{34}$

Methodological factors refer to how the study and its measurements were conducted. These include assessment effects, which may activate patients, ${ }^{36} 38-40$ contamination of health providers attending to both intervention and control arms, ${ }^{29} 38$ poor outcome fit, lack of fit between construct and measurement, or timing of measurements. ${ }^{30} 3435$ Two studies relying on documentation of $\mathrm{ADs}$ and $\mathrm{ACP}$ conversations for outcome assessment revealed clinic-level differences in the accessibility of ADs in the patient health record ${ }^{31}$ and potential registration bias in the medical file. ${ }^{43}$

\section{DISCUSSION}

This scoping review examined 16 RCTs of complex ACP interventions for patients with chronic illness, finding results that affirm the heterogeneity of intervention components, mechanisms and outcomes identified in the existing literature. ${ }^{3} 5710$

In the retained studies, outcomes across nine categories pertained to ACP actions such as completing an $\mathrm{AD}$, having consultations, patients stating their treatment preferences or SDMs judging the treatment preferences of the patient, or utterances regarding shared decision making and ACP topics during consultations. 
Outcomes related to patient well-being included quality of life measures as well as psychospiritual well-being. One study reported on a care utilisation outcome, namely place of death. Additional outcomes included decisional conflict and patient activation. Interventions were expected to influence these outcomes through mechanisms which could be grouped into seven descriptive themes, with an apparent overall emphasis on a tailored approach which facilitates understanding about the patient's illness and treatment options, encourages the patient to reflect on their personal values, and encourages the patient to talk about these topics with their carers and health providers. In order to proceed with these conversations, interventions may also encourage patients and health providers to see the importance of ACP and should equip them with the skills and confidence necessary to have these discussions effectively. Of these, the mechanism of intervention tailoring stood out as being linked to the greatest variety of outcomes in the included studies. Promoting ACP skills, confidence and competence was the mechanism most often explicitly linked to an outcome.

A finding of the present review which has not yet been fully described elsewhere is that a portion of the studies retained for full-text screening did not specify how the intervention was expected to achieve its primary outcomes: a total of 17 of 111 studies were excluded specifically for this reason.

While the application of the inclusion criteria meant that all included studies described how the intervention was expected to work or outcomes were expected to be achieved, not every study specified the mechanisms for each included component or the mechanism to which a particular outcome was expected to respond. Specifically, the anticipated impact of informational materials, ${ }^{34}$ 37-39 the provision or completion of an AD form, 282931323441 and the provision of the completed $\mathrm{AD}$ or conversation summary to a health provider or the upload thereof in the patient's medical record $^{28} 29313235$ was most often not explained by the authors. The mechanisms by which decisional conflict, ${ }^{3035} \mathrm{AD} / \mathrm{DNR}$ completion, ${ }^{31} 37$ dyad congruence, ${ }^{35}$ psychospiritual well-being ${ }^{35}$ and the number of consultations with palliative care ${ }^{41}$ were expected to change were likewise not always specified. In the one study to include place of death as a primary outcome, the authors did not state through which mechanism this outcome was expected to change; outcome selection was based on the finding that patients prefer to avoid hospital death. ${ }^{37}$

Outcomes are measured after the implementation of an intervention, in all its complexity, and components of such complex interventions may be synergistic. ${ }^{394044}$ This makes it difficult to disentangle to which degree individual components contributed to the achieved outcomes, ${ }^{37}$ and we cannot make decisive statements regarding which components of ACP do or do not work. This is, however, not the intention of this scoping review. What we do find is that the contributions of intervention components such as informational materials, $\mathrm{AD}$ forms and sharing of conversation summaries or ADs with health providers-that is, the 'how' and 'why' of these components-are often not fully explained in the present literature. The linkage of the identified mechanisms to specific outcomes similarly lacks clarity. This may make it more difficult to ascertain what the 'active ingredients' of these complex interventions are and how they work, especially in the absence of a process evaluation. ${ }^{20}$

Which outcomes ACP interventions can or should result in is still a topic of discussion. Even in this sample of studies that is smaller than that of a recent scoping review of ACP trials, ${ }^{10}$ we found a heterogeneous selection of primary outcomes. Although a Delphi panel ranked care concordance with patient wishes highest in a list of outcomes that define successful $\mathrm{ACP},{ }^{15}$ no included study featured it as a primary outcome. This outcome may require intermediate steps, such as patients being able and willing to talk about ACP, having discussions to make their wishes known to health providers and said wishes being documented. Other outcomes, such as quality of life, care and dying, may be too distant to be achieved by an ACP intervention alone, lying above the 'ceiling of accountability' for such interventions. ${ }^{45}$ Additionally, when trials examine outcomes pertaining to actions such as the occurrence of a consultation, it may be equally important to consider the quality of such consultations and the attention that is paid during these consultations to patients' values and concerns. ${ }^{46}$

Given the ongoing debate regarding ACP as a process and the outcomes which should be used to measure 'successful' ACP, ${ }^{15-17}$ these findings, viewed together, are indicative of a gap in how ACP interventions are currently reported and a point of consideration for future intervention research endeavours, from intervention development to implementation and assessment. Here, we echo the statement of Lin et $a l^{21}$ that identifying causality between the components of an intervention and the outcomes which are chosen to evaluate its effectiveness is a crucial element of publishing intervention research. Greater attention should be paid both to robust intervention development which articulates how the intervention will effect change and to clarity in reporting these mechanisms. 21224

The present scoping review lends insight into the rationales presented for outcome choices in ACP intervention trials, but also identifies potential problems in the implementation and evaluation of interventions. Even if an intervention is clear in how it is proposed to work, it may face problems during implementation: a mismatch between intervention and target population, implementation setting or the methodology of the trial. ${ }^{48}$ An absence of significant findings may indicate the intervention does not work as hypothesised or 
may point to 'teething problems' in implementation. ${ }^{20}$ The impact of ACP on the aggressiveness of care at the end of life, for instance, may be moot in settings where overtreatment or aggressive end-of-life care is not the norm ${ }^{34}$; the impact of an intervention may be limited when patients with chronic life-limiting illness are too unwell to make use of the intervention-or simply do not know how to navigate it. ${ }^{33}$ These are, ideally, factors which researchers should visualise before proceeding with an implementation study, for instance through consultation with key stakeholders. ${ }^{49}$ Of note are several interventions from the current scoping review which do precisely this, for example by taking into consideration the impact of racial disparities and then tailoring the approach of the intervention to the needs of the target population. ${ }^{31-333640}$ Finally, factors which are found in one study to impact intervention results, such as lower patient motivation to engage in ACP behaviours, have the potential to be addressed through components of interventions identified in other studies, which, for example, work by building competence and confidence and by encouraging participants to recognise the relevance of ACP.

\section{Strengths and limitations}

This scoping review is, to our knowledge, the first review to assess in RCTs of ACP interventions for chronic life-limiting illness the ways in which outcomes are expected to be achieved. By evaluating the linkage between intervention components, mechanisms and outcomes, we were also able to highlight gaps in research reporting, be it in the link between component and mechanism, or mechanism and primary outcome. Taking into account factors described by authors to explain success or failure to achieve the intended outcomes lays bare important considerations that should be made ahead of time when conducting ACP research, such as attention for the target population, the implementation of the intervention in a given setting and methodological factors. This scoping review can serve as a springboard for future realist reviews and evaluations of ACP interventions where cross-sectional, qualitative and grey literature are also included. ${ }^{50}$

Data charting for this study was done largely by one author (JS) after cross-checking a sample of extractions with a second author (ADV) and discussing the information to be extracted with all authors. The synthesis of results was performed by one author (JS). Findings were regularly discussed with other members of the research team. The framework for data charting and overall synthesis was discussed during multiple meetings with all authors, and the first and last authors conducted weekly meetings during which findings could be discussed. While we chose to include only RCTs, we did not assess the risk of bias for these studies, consistent with scoping review methodology. Further, we only included trials which involved adult patients with chronic life-limiting illness. Interventions for community-dwelling adults or populations of paediatric patients with chronic life-limiting illness may yield different findings.

\section{CONCLUSION}

This scoping review identified components of complex ACP interventions for chronic serious illness, as well as seven mechanisms by which nine outcome categories were proposed to be affected by these interventions. In reporting primary effectiveness in RCT studies, the mechanisms by which the intervention is anticipated to impact the chosen outcomes are, however, frequently unstated or unclearly stated; reporting emphasises 'what' is being done but less frequently refers to 'why' this is being done. Mechanisms such as promoting ACP skills, confidence and motivation, as well as allowing for intervention tailoring to the patient's needs, come to the fore most clearly from this review. ACP interventions should take into account patients' illness experience, willingness to engage in ACP and broader cultural considerations; these should ideally be considered during development to allow for an intervention that is sensitive and responsive to these factors. So too should researchers consider the setting in which the intervention is implemented and how the study and its measurements are conducted. In the light of recent debates regarding what outcomes ACP can be expected to achieve, this review indicates, in sum, ACP interventions do have potential to improve outcomes, but researchers should be clear in how they anticipate these outcomes will be achieved and consider the context in which these interventions are implemented.

Acknowledgements We would like to thank Veerle Piette, MSc (Ghent University) for her input regarding review methodologies and Dr Fien Mertens (Ghent University) for her insights which helped to tighten the focus of this review.

Contributors All authors contributed to the conception of the study and the formulation of its research questions. JS conducted the searches. Eligible studies were identified by JS with support from ADV and KP. Data extraction was performed by JS and checked by ADV. JS analysed the data and drafted the manuscript. All authors reviewed the manuscript, provided feedback during multiple rounds of discussion and approved the final draft. ADV and KP share last authorship.

Funding This work was supported by a predoctoral scholarship from Research Foundation - Flanders (Belgium) (Fonds Wetenschappelijk Onderzoek, FWO) (registration number $11 \mathrm{~B} 6220 \mathrm{~N})$. KP holds an FWO grant (registration number G061118N).

Disclaimer The funder has no role in the conception of the study design; in the collection, management, analysis and interpretation of data; in the writing of the manuscript; or in the decision to submit the manuscript for publication.

Competing interests None declared.

Patient consent for publication Not required.

Provenance and peer review Not commissioned; internally peer reviewed.

Open access This is an open access article distributed in accordance with the Creative Commons Attribution Non 
Commercial (CC BY-NC 4.0) license, which permits others to distribute, remix, adapt, build upon this work noncommercially, and license their derivative works on different terms, provided the original work is properly cited, appropriate credit is given, any changes made indicated, and the use is noncommercial. See: http://creativecommons.org/licenses/by-nc/4. $0 /$.

\section{ORCID iD}

Julie Stevens http://orcid.org/0000-0002-1417-0436

\section{REFERENCES}

1 Rietjens JAC, Sudore RL, Connolly M, et al. Definition and recommendations for advance care planning: an international consensus supported by the European association for palliative care. Lancet Oncol 2017;18:e543-51.

2 Sudore RL, Fried TR. Redefining the "planning" in advance care planning: preparing for end-of-life decision making. Ann Intern Med 2010;153:256-61.

3 Houben CHM, Spruit MA, Groenen MTJ, et al. Efficacy of advance care planning: a systematic review and meta-analysis. $J$ Am Med Dir Assoc 2014;15:477-89.

4 Fahner JC, Beunders AJM, van der Heide A, et al. Interventions guiding advance care planning conversations: a systematic review. J Am Med Dir Assoc 2019;20:227-48.

5 Brinkman-Stoppelenburg A, Rietjens JAC, van der Heide A. The effects of advance care planning on end-of-life care: a systematic review. Palliat Med 2014;28:1000-25.

6 Weathers E, O'Caoimh R, Cornally N, et al. Advance care planning: a systematic review of randomised controlled trials conducted with older adults. Maturitas 2016;91:101-9.

7 Jimenez G, Tan WS, Virk AK, et al. Overview of systematic reviews of advance care planning: summary of evidence and global lessons. J Pain Symptom Manage 2018;56:e25:436-59.

8 Howard M, Bonham AJ, Heyland DK, et al. Measuring engagement in advance care planning: a cross-sectional multicentre feasibility study. BMJ Open 2016;6:e010375-7.

9 Jimenez G, Tan WS, Virk AK, et al. State of advance care planning research: a descriptive overview of systematic reviews. Pall Supp Care 2019;17:234-44.

10 McMahan RD, Tellez I, Sudore RL. Deconstructing the complexities of advance care planning outcomes: what do we know and where do we go? A scoping review. J Am Geriatr Soc 2021;69:234-44.

11 Fleuren N, Depla MFIA, Janssen DJA, et al. Underlying goals of advance care planning (ACP): a qualitative analysis of the literature. BMC Palliat Care 2020;19:1-15.

12 Moody SY. "Advance" care planning reenvisioned. J Am Geriatr Soc 2021;69:330-2.

13 Gilissen J, Van den Block L, Pivodic L. Complexities and outcomes of advance care planning. JAMA Intern Med 2021;181:142-3.

14 Biondo PD, Lee LD, Davison SN, et al. How healthcare systems evaluate their advance care planning initiatives: results from a systematic review. Palliat Med 2016;30:720-9.

15 Sudore RL, Heyland DK, Lum HD, et al. Outcomes that define successful advance care planning: a delphi panel consensus. $J$ Pain Symptom Manage 2018;55:245-55.

16 Sean Morrison R. Advance Directives/Care planning: clear, simple, and wrong. J Palliat Med 2020;23:878-9.

17 Tishelman C, Eneslätt M, Menkin ES, et al. Tishelman et al's response to Morrison: advance Directives/Care planning: clear, simple, and wrong (DOI: 10.1089/jpm.2020.0272). J Palliat Med 2021;24:16-17.

18 Lum HD, Barnes DE, Katen MT, et al. Improving a full range of advance care planning behavior change and action domains: the prepare randomized trial. J Pain Symptom Manage 2018;56:575-81.

19 Rietjens J, Korfage I, van der Heide A. Advance care planning: not a panacea. Palliat Med 2016;30:421-2.
20 Craig P, Dieppe P, Macintyre S, Michie S, Nazareth I, Petticrew $\mathrm{M}$. Developing and evaluating complex interventions: new guidance Medical Research Council; 2019. https://mrc.ukri. org/documents/pdf/complex-interventions-guidance/

21 Lin C-P, Evans CJ, Koffman J, et al. The conceptual models and mechanisms of action that underpin advance care planning for cancer patients: a systematic review of randomised controlled trials. Palliat Med 2019;33:5-23.

22 Hoffmann TC, Glasziou PP, Boutron I, et al. Better reporting of interventions: template for intervention description and replication (TIDieR) checklist and guide. $B M J$ 2014;348:g1687.

23 Montgomery C, Hickman SE, Wilkins C, et al. Montgomery et al's response to morrison: advance directives/care planning: clear, simple, and wrong (DOI: 10.1089/jpm.2020.0272).J Palliat Med 2021;24:12-13.

24 Munn Z, Peters MDJ, Stern C, et al. Systematic review or scoping review? guidance for authors when choosing between a systematic or scoping review approach. BMC Med Res Methodol 2018;18:143.

25 Arksey H, O'Malley L. Scoping studies: towards a methodological framework. Int J Soc Res Methodol $2005 ; 8: 19-32$.

26 Tricco AC, Lillie E, Zarin W, et al. PRISMA extension for scoping reviews (PRISMA-ScR): checklist and explanation. Ann Intern Med 2018;169:467-73.

27 Levac D, Colquhoun H, O'Brien KK. Scoping studies: advancing the methodology. Implementation Sci 2010;5:69.

28 Brazil K, Carter G, Cardwell C, et al. Effectiveness of advance care planning with family carers in dementia nursing homes: a paired cluster randomized controlled trial. Palliat Med 2018;32:603-12.

29 Doorenbos AZ, Levy WC, Curtis JR, et al. An intervention to enhance goals-of-care communication between heart failure patients and heart failure providers. J Pain Symptom Manage 2016;52:353-60.

30 Hilgeman MM, Allen RS, Snow AL, et al. Preserving identity and planning for advance care (PIPAC): preliminary outcomes from a patient-centered intervention for individuals with mild dementia. Aging Ment Health 2014;18:411-24.

31 Lyon ME, Squires L, D'Angelo LJ, et al. Family-centered (face) advance care planning among African-American and NonAfrican-American adults living with HIV in Washington, DC: a randomized controlled trial to increase documentation and health equity. J Pain Symptom Manage 2019;57:607-16.

32 Lyon ME, Squires L, Scott RK, et al. Effect of family centered (FACE $®)$ advance care planning on longitudinal congruence in end-of-life treatment preferences: a randomized clinical trial. AIDS Behav 2020;24:3359-75.

33 Nedjat-Haiem FR, Cadet TJ, Amatya A, et al. Efficacy of motivational interviewing to enhance advance directive completion in Latinos with chronic illness: a randomized controlled trial. Am J Hosp Palliat Care 2019;36:980-92.

34 Overbeek A, Korfage IJ, Jabbarian LJ, et al. Advance care planning in frail older adults: a cluster randomized controlled trial. J Am Geriatr Soc 2018;66:1089-95.

35 Song M-K, Donovan HS, Piraino BM, et al. Effects of an intervention to improve communication about end-of-life care among African Americans with chronic kidney disease. Appl Nurs Res 2010;23:65-72.

36 Song M-K, Ward SE, Fine JP, et al. Advance care planning and end-of-life decision making in dialysis: a randomized controlled trial targeting patients and their surrogates. Am J of Dis 2015;66:813-22.

37 Stein RA, Sharpe L, Bell ML, et al. Randomized controlled trial of a structured intervention to facilitate end-of-life decision making in patients with advanced cancer. J Clin Oncol 2013;31:3403-10. 
38 Walczak A, Butow PN, Tattersall MHN, et al. Encouraging early discussion of life expectancy and end-of-life care: a randomised controlled trial of a nurse-led communication support program for patients and caregivers. Int J Nurs Stud 2017;67:31-40.

39 Sudore RL, Boscardin J, Feuz MA, et al. Effect of the prepare website vs an easy-to-read advance directive on advance care planning documentation and engagement among Veterans. JAMA Intern Med 2017;177:1102-9.

40 Sudore RL, Schillinger D, Katen MT. Engaging diverse Englishand Spanish-speaking older adults in advance care planning. JAMA Intern Med 2018;94121:1-10.

41 Vogel RI, Petzel SV, Cragg J, et al. Development and pilot of an advance care planning website for women with ovarian cancer: a randomized controlled trial. Gynecol Oncol 2013;131:430-6.

42 Goossens B, Sevenants A, Declercq A, et al. Improving shared decision-making in advance care planning: implementation of a cluster randomized staff intervention in dementia care. Patient Educ Couns 2020;103:839-47.

43 Tilburgs B, Koopmans R, Vernooij-Dassen M, et al. Educating Dutch general practitioners in dementia advance care planning: a cluster randomized controlled trial. J Am Med Dir Assoc 2020;21:837-42.
44 Schichtel M, Wee B, Perera R, et al. Clinician-targeted interventions to improve advance care planning in heart failure: a systematic review and meta-analysis. Heart 2019;105:1316-24.

45 Gilissen J, Pivodic L, Gastmans C, et al. How to achieve the desired outcomes of advance care planning in nursing homes: a theory of change. BMC Geriatr 2018;18:1-14.

46 Tulsky JA, Fischer GS, Rose MR, et al. Opening the black box: how do physicians communicate about advance directives? Ann Intern Med 1998;129:441-9.

47 Davidoff F, Dixon-Woods M, Leviton L. Demystifying theory and its use in improvement. BMJ Qual bamp 2015;24:228-38.

48 Kumpunen S, Edwards N, Georghiou T. Why are evaluations not producing the results we expect? Int J Care Coord 2019;1:21.

49 O'Cathain A, Croot L, Duncan E, et al. Guidance on how to develop complex interventions to improve health and healthcare. BMJ Open 2019;9:e029954.

50 Pawson R, Greenhalgh T, Harvey G, et al. Realist review-a new method of systematic review designed for complex policy interventions. J Health Serv Res Policy 2005;10 Suppl $1: 21-34$. 